The First Affiliated Hospital of Guangxi Medical University, Nanning Guangxi, People's Republic of China

doi:10.1016/j.jtcvs.2010.12.009

\section{ADVANTAGES OF THE CONTINUOUS TELESCOPIC INVERSION TECHNIQUE DO NOT OVERCOME THE DISADVANTAGES OF THE "TURN-UP" TECHNIQUE OF AORTIC ANASTOMOSIS \\ To the Editor:}

Rylski and associates ${ }^{1}$ should be congratulated for the favorable outcomes of the continuous telescopic anastomosis technique applied for a diseased aorta and Dacron prosthesis. They mentioned that their technique is superior to our "turn-up" anastomosis method (2-layer anastomosis involving 6 to 8 pairs of firstline everting $\mathrm{U}$ stay sutures followed by second-line continuous over-andover sutures), ${ }^{2}$ because they believe their method requires less time and does not have a ridge projecting into the bloodstream. We wish to correct their misunderstanding, explain what defines good anastomosis to the readers of the Journal, and present the advantages of the turn-up method.
In the technique reported by Rylski and colleagues, external felt is placed separately from the sutures, which takes additional time. However, in the turn-up method, external felt for strengthening the distal aorta is simultaneously placed with 6 to $8 \mathrm{U}$ stay sutures, which are placed for everting 3 to $5 \mathrm{~mm}$ of prosthetic edge in preparation for the second layer of anastomosis. In this sense, both methods require 2 layers for complete anastomosis. However, we do not recommend reducing the number of first-line $U$ stay sutures to save time; at least 6 pairs are necessary for effective eversion.

With regard to technical difficulty, in the method described by Rylski and associates, the suture goes out-in the aorta, then out-in and in-out the prosthesis, which indicates that the surgeon has to frequently change the direction of the needle. This complexity may prevent others from using this technique for the distal aorta, particularly near the bronchial artery, because surgeons find it difficult to place the sutures at the nadir of the deep arterial valley. In fact, they predominantly use this technique for the proximal aorta. Meanwhile, the turnup method involves only forehand suturing and can be used regardless of the position of the anastomosis, that is, proximal, distal, or very distal (Figure 1). Moreover, because the first-line sutures are carefully placed separately, adventitia is not missed even with very diseased aortas, and it is much easier to place second-line sutures by retracting the prosthesis and grasping the everted portion.

With regard to hemostatic power, the technique of Rylski and associates basically involves 1-layer continuous sutures, with the prosthesis placed inside the aorta; thus, hemostasis is achieved by a single suture line. In the turn-up method, second-line continuous over-and-over sutures are placed along the everted portion, and hemostasis is achieved by a suture band 3 to $5 \mathrm{~mm}$ in circumference, which seems more stable. Moreover, even if anastomotic bleeding occurs, additional sutures can easily be placed along the everted prosthesis and aortic wall.

From January 2003 to October 2010, our turn-up technique was used for 440 aortic aneurysm cases and 170 Stanford A aortic dissection cases. Although needle hole bleeding from the fragile dissected aorta occurred occasionally, there has been no re-exploration for bleeding from the anastomosis and no postoperative false aneurysms occurred due to this technique. Further, no ridge because
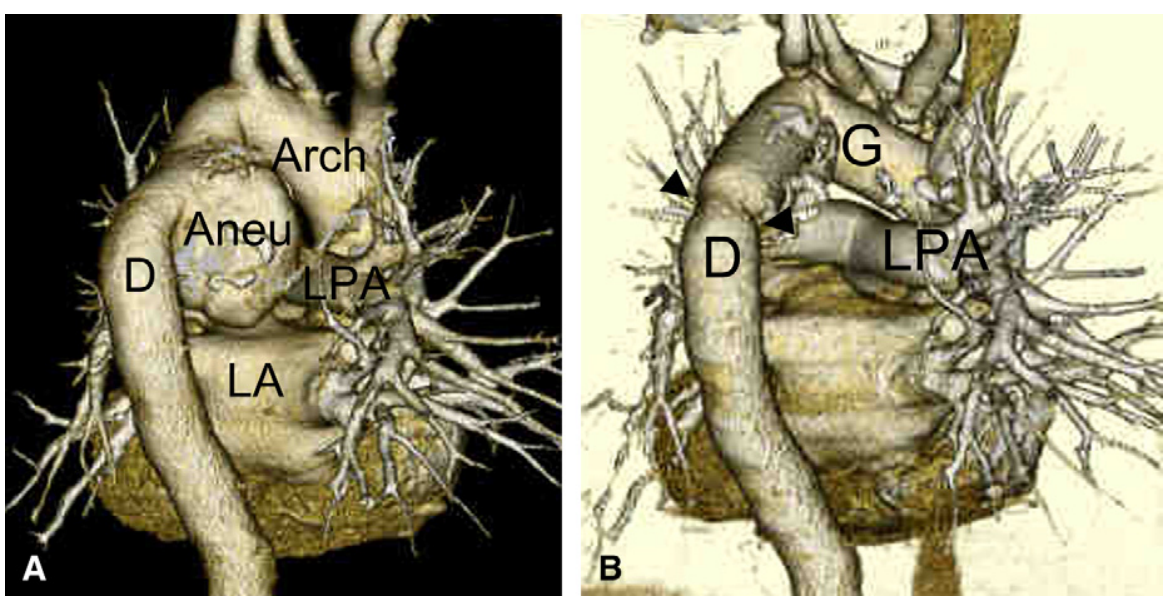

FIGURE 1. A, Preoperative 3-dimensional computed tomographic image in the dorsal view of a patient with a huge distal arch aneurysm; the diameter of the aneurysm was normalized behind the bifurcation of the pulmonary artery. B, Postoperative image of the same patient as in A. The arch and proximal descending aorta were completely replaced with a prosthesis (arrowhead) placed through a median sternotomy without any additional incision. Arch, Aortic arch; Aneu, aneurysm; $L P A$, left pulmonary artery; $D$, Descending aorta; $L A$, Left atrium. B, $G$, prosthetic graft. 
of the everted prosthesis or postoperative hemostasis associated with anastomotic distortion was noted. Thus, the effectiveness of the turn-up method was confirmed with a significantly sized patient cohort and suitable follow-up period.

\section{Takeshi Shimamoto, $M D, P h D$ \\ Tatsuhiko Komiya, MD \\ Department of Cardiovascular Surgery \\ Kurashiki Central Hospital Okayama, Japan}

\section{References \\ 1. Rylski B, Siepe M, Schoellhorn J, Beyersdorf F. An improved technique for aortic anastomosis: graft telescopic inversion. J Thorac Cardiovasc Surg. 2010;140:934-5. \\ 2. Tamura N, Komiya T, Sakaguchi G, Kobayashi T 'Turn-up' anastomotic technique for acute aortic dissection. Eur J Cardiothorac Surg. 2007;31: 548-9.}

doi:10.1016/j.jtcvs.2010.11.047

\section{Reply to the Editor:}

We read with interest the letter of Shimamato and Komiya comparing our novel technique for aortic anastomosis using telescopic graft inversion ${ }^{1}$ with the "turn-up" method. ${ }^{2}$ We appreciate all thoughtful comments and would like to address some of the important points involved.

First of all, Shimamato and Komiya discussed the time needed for the 2 suture techniques. Both anastomotic techniques are time-consuming. We reported that our method may take about $40 \%$ longer than the simple "over-and-over" technique. Both "turn-up" and graft telescopic inversion methods involve 2 layers of sutures for complete anastomosis. However, the easy mattress suture of felt strip in our technique extended the time for anastomosis only slightly. Additionally, there was no need for hemostatic stitches after completing the anastomosis, which might save time. Our method thus reduced the total time for the procedure significantly, mainly because we needed less time to stop the bleeding. We are convinced that the addition of the technique of Shimamato and Komiya in selected cases can save overall time and morbidity as well.

In describing our method, we concluded that there is no ridge in the bloodstream in comparison with the "turn-up" method. Inverted Dacron aortic anastomosis results in 1 layer of intraluminal Dacron at the anastomosis level, whereas the "turn-up" technique requires 2 intraluminal layers, which may result in a ridge projecting into the bloodstream. We believe that an anastomotic stenosis or any intraluminal edge can be unfavorable and elicit embolic events. With our method, we try to prevent anastomotic stenosis by completing the anastomosis first, then opening the crossclamp, and carefully tightening and knotting the suture for the external felt with full pressure filling to prevent suspected tourniquet syndrome on the anastomotic side.

We are convinced that both techniques are appropriate for aortic replacement, especially when the arterial wall is highly fragile, as in aortic dissection or in patients with Marfan syndrome. Please allow us to recommend our technique as a worthwhile supplement to the surgical armamentarium that can be used in cases such as those mentioned above.

Bartosz Rylski, MD

Matthias Siepe, $M D$

Joachim Schoellhorn, MD

Friedhelm Beyersdorf, MD, PhD

University Cardiovascular Center

Freiburg-Bad Krozingen

Freiburg, Germany

\section{References}

1. Rylski B, Siepe M, Schoellhorn J, Beyersdorf F. An improved technique for aortic anastomosis: telescopic graft inversion. $J$ Thorac Cardiovasc Surg. 2010;140:934-5

2. Tamura N, Komiya T, Sakaguchi G, Kobayashi T. "Turn-up" anastomotic technique for acute aortic dissection. Eur J Cardiothorac Surg. 2007;31: 548-9.

doi:10.1016/j.jtcvs.2010.12.016

\section{IMMORTAL PERSON-TIME BIAS IN OBSERVATIONAL STUDIES IN CARDIAC SURGERY \\ To the Editor:}

We read with great interest the article by Kim and associates ${ }^{1}$ in a recent issue of the Journal of Thoracic and Cardiovascular Surgery. We find this topic area engaging and relevant to clinical practice, especially in light of expanding indications of dual antiplatelet therapy and recent advances in perioperative management lending to improved acute operative mortality. ${ }^{2}$ However, we have important concerns regarding the methodology and statistical analyses undertaken.

The investigators compared the treatment strategies of aspirin plus clopidogrel with aspirin alone in patients undergoing isolated coronary artery bypass grafting (CABG). Dual therapy was associated with a $50 \%$ risk reduction (odds ratio [OR], 0.50; $95 \%$ confidence interval $[\mathrm{CI}], 0.25-$ 0.99 ) in in-hospital mortality, a $30 \%$ risk reduction $(\mathrm{OR}, 0.70 ; 95 \% \mathrm{CI}$, 0.51-0.97) in bleeding events, and no effect on ischemic/thrombotic events (OR, 0.99; 95\% CI, 0.591.64). These robust, and seemingly contradictory, findings may be influenced by important biases.

First, the exposure measurement was based on the addition of clopidogrel in days 1 and 2 postoperatively, with primary outcome assessment beginning immediately after the operation. Patients must thus survive (ie, are "immortal") those days into the postoperative period to be defined as exposed to the clopidogrel therapy, whereas patients experiencing an adverse event previously were thus necessarily included in the unexposed group (aspirin alone). Such a classification of exposure to clopidogrel leads to immortal time bias. ${ }^{3}$ The magnitude of this bias is directly related to the proportion of length of stay (not provided in text) that is immortal, with shorter length of stays resulting in higher bias, and the number 